Check for updates

Cite this: RSC Adv., 2019, 9, 20925

\section{Theoretical calculation of a full-dimensional $a b$ initio potential energy surface and prediction of infrared spectra for $\mathrm{Xe}-\mathrm{CS}_{2}$}

\begin{abstract}
Miao Qin,,$^{\mathrm{ab}}$ Xiuchan Xiao (D) $\dagger^{\star a b}$ and Hua Zhu ${ }^{\mathrm{c}}$
An effective four-dimensional (4D) ab initio potential energy surface (PES) for $\mathrm{Xe}-\mathrm{CS}_{2}$ which explicitly involves the intramolecular $Q_{1}$ symmetric stretching and $Q_{3}$ antisymmetric stretching vibrational coordinates of $\mathrm{CS}_{2}$ is constructed. The computations are carried out employing single- and doubleexcitation coupled-cluster theory with a non-iterative perturbation treatment of triple excitations [CCSD(T)] method with a large basis set. Two vibrationally averaged potentials at the ground and $\nu_{1}+\nu_{3}$ $\left(\nu_{1}=1, \nu_{3}=1\right)$ excited states are obtained by integrating the $4 \mathrm{D}$ potentials over the $Q_{1}$ and $Q_{3}$ coordinates. The potentials have a T-shaped global minimum and two equivalent linear local minima. The radial discrete variable representation/angular finite basis representation and the Lanczos algorithm are employed to calculate the rovibrational energy levels for $\mathrm{Xe}-\mathrm{CS}_{2}$. The infrared band origin shift associated with the fundamental band of $\mathrm{CS}_{2}$ is predicted, which is red-shifted by $-1.996 \mathrm{~cm}^{-1}$ in the $\nu_{1}+\nu_{3}$ region. In addition, we further predict the spectroscopic parameters for the ground and the $\nu_{1}+$ $\nu_{3}$ excited states of $\mathrm{Xe}-\mathrm{CS}_{2}$. Compared with the previous $\mathrm{Rg}-\mathrm{CS}_{2}(\mathrm{Rg}=\mathrm{He}, \mathrm{Ne}, \mathrm{Ar}, \mathrm{Kr})$ complexes, we found that the complexes of the rare gas atoms with $\mathrm{CS}_{2}$ display obvious regularities.
\end{abstract}

Received 20th May 2019

Accepted 17th June 2019

DOI: 10.1039/c9ra03782a

rsc.li/rsc-advances spectroscopic parameters were presented. Theoretically, a number of ab initio potential energy surfaces (PESs) for Rg$\mathrm{CS}_{2}$ were constructed at different levels of theory. For instance, Farrokhpour and co-workers ${ }^{27}$ first determined the PESs for the $\mathrm{Rg}-\mathrm{CS}_{2}$ complexes using $\mathrm{CCSD}(\mathrm{T})$ theory with the aug-cc-pVDZ basis set. Soon after, Zang et al. ${ }^{28}$ reported the twodimensional PES for the $\mathrm{Rg}-\mathrm{CS}_{2}(\mathrm{Rg}=\mathrm{He}, \mathrm{Ne}, \mathrm{Ar})$ complexes by using the aug-cc-pVTZ basis set at the $\operatorname{CCSD}(\mathrm{T})$ level. However, these theoretical studies have obtained the PESs that encompass only two intermolecular vibration coordinates and a fixed $\mathrm{CS}_{2}$ geometry. The results showed that the spectral pattern in the infrared region cannot be properly identified. Thus, construction of the PESs that involve the intramolecular vibration modes of $\mathrm{CS}_{2}$ is needed. Recently, we calculated a 3D PES of the $\mathrm{Rg}-\mathrm{CS}_{2}$ complex $^{\mathbf{2 9}, 30}$ including the antisymmetric stretching $Q_{3}$ coordinate of the $\mathrm{CS}_{2}$ monomer, and showed a good agreement with the experimental infrared data. In order to explore more detailed spectral information for $\mathrm{Rg}-\mathrm{CS}_{2}$, we further carried out the calculation of a four-dimensional PES of the $\mathrm{Rg}-\mathrm{CS}_{2}$ (ref. 31-33) complexes, which incorporates the $Q_{1}$ symmetric and $Q_{3}$ anti-symmetric stretching coordinates of $\mathrm{CS}_{2}$. We have successfully reported the 4D PESs for the complexes of the lighter rare gas atoms with $\mathrm{CS}_{2}$ by this method. To our knowledge, the experimental and theoretical information in the $\nu_{1}+\nu_{3}$ region are absent for $\mathrm{Xe}-\mathrm{CS}_{2}$.

In order to discover the trends and diversities among the $\mathrm{Rg}$ $\mathrm{CS}_{2}$ complexes, we present a reliable $4 \mathrm{D}$ PES for Xe-CS $\mathrm{CS}_{2}$ with $\mathrm{CS}_{2}$ in the $\nu_{1}+\nu_{3}$ region by applying the $\operatorname{CCSD}(\mathrm{T})$ method with the

\footnotetext{
${ }^{a}$ School of Architectural and Environmental Engineering, Chengdu Technological University, Chengdu 611730, China. E-mail: shawailsa@sina.cn

${ }^{b}$ Center of Big Data for Smart Environmental Protection, Chengdu Technological University, Chengdu 611730, China

'School of Chemistry, Sichuan University, Chengdu 610064, China

$\dagger$ Miao Qin and Xiuchan Xiao contributed equally to this work.
} 
aug-cc-pVQZ basis set. This paper is arranged as follows: in Section II, we show the computational details that include $a b$ initio and rovibrational energy levels. The discussion of the PES, the calculated rovibrational bound states, and the predicted infrared spectra are presented in Sections III-IV. Finally, a brief conclusion is given in Section $\mathrm{V}$.

\section{Computational details}

\section{A. Ab initio calculations}

For the $\mathrm{Xe}-\mathrm{CS}_{2}$ dimer, the Jacobi coordinates $\left(R, \theta, Q_{1}, Q_{3}\right)$ are employed to describe the geometry. In the geometric variables, $R$ denotes the intermolecular distance between the center of mass of $\mathrm{CS}_{2}$ to the Xe atom, the angle of the vector $R$ with respect to the $\mathrm{CS}_{2}$ molecule is defined as $\theta . Q_{1}$ and $Q_{3}$ are the normal mode coordinates, which describe the $\nu_{1}$ symmetric stretching vibration and $\nu_{3}$ antisymmetric stretching vibration of $\mathrm{CS}_{2}$, respectively. Here, the intramolecular vibrational coordinates $\left(Q_{1}, Q_{3}\right)$ can be simply defined as

$$
\begin{gathered}
Q_{1}=\left(r_{\mathrm{CS}_{1}}+r_{\mathrm{CS}_{2}}-2 r_{\mathrm{e}}\right) / \sqrt{2} \\
Q_{3}=\left(r_{\mathrm{CS}_{1}}-r_{\mathrm{CS}_{2}}\right) / \sqrt{2}
\end{gathered}
$$

where $r_{\mathrm{CS}_{1}}$ and $r_{\mathrm{CS}_{2}}$ mean the two $\mathrm{C}-\mathrm{S}$ bond lengths of $\mathrm{CS}_{2}, r_{\mathrm{e}}$ is the average bond length derived from experimental spectra data. ${ }^{34}$ The two-dimensional $Q_{1}$ and $Q_{3}$ potential curves were computed at the $\operatorname{CCSD}(\mathrm{T})$ level to determine the energy levels and wave functions for the $Q_{1}$ and $Q_{3}$ modes. The coordinate scaling method $^{35}$ was employed to adjust the two-dimensional potential in order to reproduce the experimental frequencies for the fundamental band. ${ }^{34}$ We generated 25 potential optimized discrete variable representation (PODVR) ${ }^{36,37}$ grid points corresponding to $Q_{1}=-0.122411,-0.044046,-0.027968,-0.101203$, $-183772 a_{0}, \quad Q_{3}=-0.245743, \quad-0.116813, \quad 0.0,0.116813$, $0.245743 a_{0}$ for the ground state, and $Q_{1}=-0.139155,-0.060791$, 0.011220, 0.084454, 0.167022 $a_{0}, Q_{3}=-0.247934,-0.117859,0.0$, $0.117859,0.247934 a_{0}$ for the $\nu_{1}+\nu_{3}\left(\nu_{1}=1, \nu_{3}=1\right)$ excited state.

The $a b$ initio potential energies were computed for a total of about 9000 discrete points. A relatively dense grid was calculated with 27 values of $R$ ranging from $5.50 a_{0}$ to $24.00 a_{0}$ and 13 points of $\theta$ from $0^{\circ}$ to $180^{\circ}$ at intervals of $15^{\circ}$. The four dimensional PES for Xe- $\mathrm{CS}_{2}$ was performed using the $\operatorname{CCSD}(\mathrm{T})^{38}$ method. The aug-cc-pVQZ basis set of Woon and Dunning ${ }^{39}$ was used for carbon and sulfur atoms, and the quasirelativistic pseudopotential aug-cc-pVQZ-PP ${ }^{40}$ basis set was used for the Xe atom, supplemented with an additional set of bond functions (3s3p2d1f1g). ${ }^{41}$ The full counterpoise procedure (FCP) ${ }^{42}$ was selected to correct the basis set superposition error (BSSE). The vibrationally averaged 2D potentials $V_{\nu_{1}+\nu_{3}}(R, \theta)$ were obtained by averaging the $4 \mathrm{D}$ potential over the $Q_{1}$ and $Q_{3}$ vibrational coordinates, which can be written as

$$
\begin{aligned}
& V_{\nu_{1}+\nu_{3}}(R, \theta)= \\
& \quad \int_{-\infty}^{\infty} \psi_{\nu_{1}+\nu_{3}}\left(Q_{1}, Q_{3}\right) V\left(R, \theta, Q_{1}, Q_{3}\right) \psi_{\nu_{1}+\nu_{3}}\left(Q_{1}, Q_{3}\right) \mathrm{d} Q_{1} \mathrm{~d} Q_{3}
\end{aligned}
$$

The cubic spline interpolation was employed to generate the averaged PESs for $R$ and $\theta$ coordinates. The root-mean-square (rms) deviation is about $0.08 \mathrm{~cm}^{-1}$ in the final fit. All calculations were carried out using the MOLPRO package. ${ }^{43}$

\section{B. Calculations of rovibrational energy levels}

With the Born-Oppenheimer approximation, the vibrational averaged 2D intermolecular Hamiltonian of the $\mathrm{Xe}-\mathrm{CS}_{2}$ complex can be written as ${ }^{\mathbf{4 4 , 4 5}}$

$$
\frac{\hat{H}=-\frac{1}{2 \mu} \frac{\partial^{2}}{\partial R^{2}}+\frac{\hat{j}^{2}}{2 I_{\nu_{1}+\nu_{3}}}+(\hat{J}-\hat{j})^{2}}{2 \mu R^{2}+V_{\nu_{1}+\nu_{3}}(R, \theta)}
$$

where $\mu$ is the reduced mass of the Xe- $\mathrm{CS}_{2}$ complex, $\hat{J}$ and $\hat{j}$ are the angular momentum operators for the total and monomer rotations. $I_{\nu_{1}+\nu_{3}}$ represents the vibrationally averaged rotational moment of the inertia of $\mathrm{CS}_{2}$, which can be defined by the following equation,

$$
I_{\nu_{1}+\nu_{3}}=\int_{-\infty}^{\infty} \psi_{\nu_{1}+\nu_{3}}\left(Q_{1}, Q_{3}\right) I_{Q_{1}+Q_{3}} \psi_{\nu_{1}+\nu_{3}}\left(Q_{1}, Q_{3}\right) \mathrm{d} Q_{1} \mathrm{~d} Q_{3}
$$

Based on the PES, the rovibrational Hamiltonian and wave functions were calculated with the radial DVR/angular FBR method. ${ }^{46,47}$ In our work, 120 sine-DVR ${ }^{48}$ grid points were used for the radial coordinate $R$. For the angular coordinate, we selected 90 DVR grids and 89 basis functions of associated Legendre polynomials. The Lanczos algorithm method ${ }^{\mathbf{4 9 5}}$ was used to diagonalize the Hamiltonian matrix, and we selected 10000 Lanczos iterations to obtain the eigenvalues and eigenvectors. For the angular part, the parity-adapted rotational basis for the three Euler angles $(\alpha, \beta, \gamma)$ can be written as

$$
\begin{aligned}
C_{K M}^{J p}(\alpha, \beta, \gamma)= & {\left[2\left(1+\delta_{K 0}\right)\right]^{-1 / 2}\left[D_{M K}^{J^{*}}(\alpha, \beta, \gamma)\right.} \\
& \left.+(-1)^{J+K+p} D_{M-K}^{J^{*}}(\alpha, \beta, \gamma)\right], \quad p=0,1
\end{aligned}
$$

where $D_{M K}^{J}(\alpha, \beta, \gamma)$ were the normalized rotational functions for $\mathrm{Xe}-\mathrm{CS}_{2}$, the space-inversion parity $p=0$ or 1 illustrates that the basis is even or odd under inversion. The total parity was given by $(-1)^{J^{+P}}$.

\section{Potential energy surface}

The contour plot of the vibrationally averaged $2 \mathrm{D} \nu_{1}+\nu_{3}$ excited state PES is showed in Fig. 1, which clearly displays that the global minimum is a T-shaped configuration at $R=3.97 \AA$ and $\theta=90.0^{\circ}$ with a well depth of $413.875 \mathrm{~cm}^{-1}$. In addition, there are two equivalent linear local minima at $R=5.56 \AA$ and $\theta=$ $0^{\circ}$ or $180^{\circ}$ with a depth of $248.045 \mathrm{~cm}^{-1}$. In our PES, between the two minima, there are two saddle points with an energy barrier of $187.997 \mathrm{~cm}^{-1}$ relative to the global minimum, located at $R=$ $5.08 \AA$ and $\theta=45^{\circ}$ or $135^{\circ}$. In order to compare the Xe-CS complex with the other $\mathrm{Rg}-\mathrm{CS}_{2}$ complexes, the differences in the geometries and well depths between the $\mathrm{Rg}-\mathrm{CS}_{2}$ complexes $^{31-33,51,52}$ are listed in Table 1 . One can see that the Rg$\mathrm{CS}_{2}$ complexes have similar shapes, such as the T-shaped global minimum, two linear local minima, and two saddle points. 


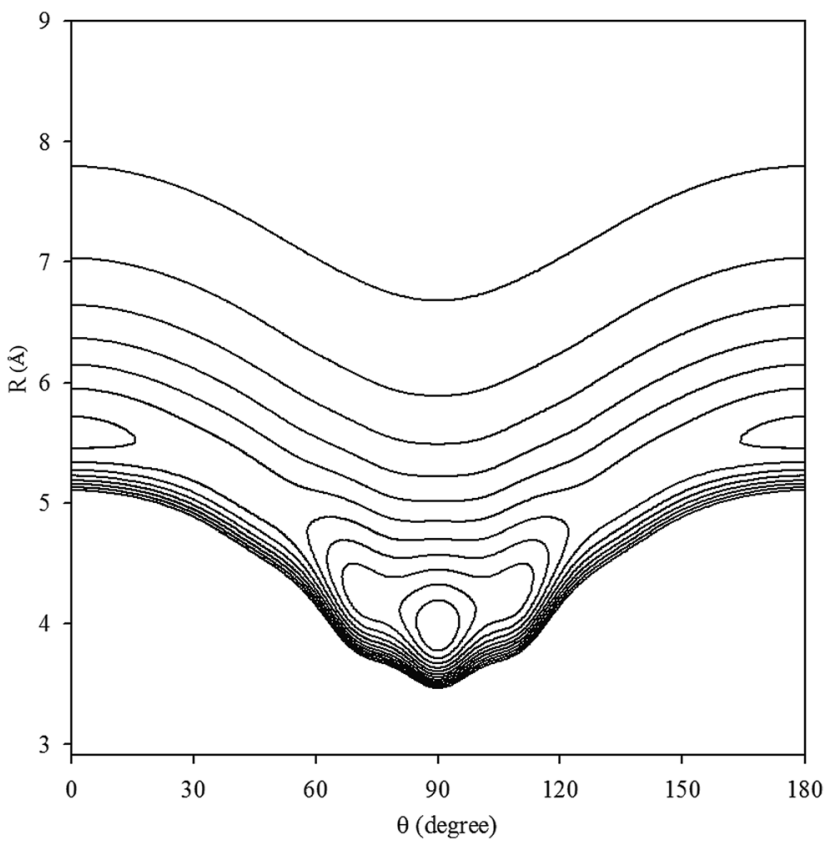

Fig. 1 Contour plot (in $\mathrm{cm}^{-1}$ ) of the averaged intermolecular potential energy surface for $\mathrm{Xe}-\mathrm{CS}_{2}$ with $\mathrm{CS}_{2}$ at the $\nu_{1}+\nu_{3}$ excited state.

However, there are some deviations among the $\mathrm{Rg}-\mathrm{CS}_{2}$ complexes. For example, the depth of the global and local minima become deeper from $\mathrm{He}-\mathrm{CS}_{2}$ to $\mathrm{Xe}-\mathrm{CS}_{2}$, which indicates the intermolecular interactions become stronger with the increasing mass of the rare gas atom. For another, the well depth of $\mathrm{Xe}-\mathrm{CS}_{2}$ is much larger than the other $\mathrm{Rg}-\mathrm{CS}_{2}$ complexes, which means the Xe atom is strongly hindered from free motion around the $\mathrm{CS}_{2}$ molecule. In addition, the minimum energy distance gradually become larger from He$\mathrm{CS}_{2}$ to $\mathrm{Xe}-\mathrm{CS}_{2}$. Compared with the previous theoretical study of the potential, ${ }^{51}$ the contour plots look almost the same as those for our work, and the positions and energies of the stationary points are shifted only slightly.

\section{Bound states of rovibrational energy levels and infrared spectra}

Based on the potential, the energy levels of the bound states were calculated. In order to simplify our writing, we label the $\nu_{1}+\nu_{3}$ of $\mathrm{CS}_{2}$ as the $\nu_{5}$ vibrational state for Xe- $\mathrm{CS}_{2}$. In Table 2, we display the first twenty pure vibrational bound states of the Xe-
Table 2 The calculated energy levels (in $\mathrm{cm}^{-1}$ ) for the first twenty vibrational bound states of $\mathrm{Xe}-\mathrm{CS}_{2}$ with $\mathrm{CS}_{2}$ at the ground and the $\nu_{1}+$ $\nu_{3}$ excited states

\begin{tabular}{|c|c|c|c|c|c|c|c|}
\hline \multicolumn{4}{|c|}{ Ground state } & \multicolumn{4}{|c|}{$\nu_{5}$ state } \\
\hline$N$ & & $N$ & & $N$ & & $N$ & \\
\hline 0 & -372.243 & 10 & -276.947 & 0 & -374.239 & 10 & -278.046 \\
\hline 1 & -340.903 & 11 & -275.624 & 1 & -342.887 & 11 & -276.971 \\
\hline 2 & -330.927 & 12 & -273.955 & 2 & -335.591 & 12 & -275.602 \\
\hline 3 & -312.666 & 13 & -266.022 & 3 & -314.627 & 13 & -266.029 \\
\hline 4 & -308.886 & 14 & -258.545 & 4 & -310.894 & 14 & -259.381 \\
\hline 5 & -308.813 & 15 & -257.516 & 5 & -310.755 & 15 & -259.247 \\
\hline 6 & -298.008 & 16 & -256.517 & 6 & -299.059 & 16 & -256.907 \\
\hline 7 & -295.275 & 17 & -252.014 & 7 & -296.373 & 17 & -253.608 \\
\hline 8 & -284.470 & 18 & -251.523 & 8 & -285.564 & 18 & -253.276 \\
\hline 9 & -283.164 & 19 & -245.973 & 9 & -284.769 & 19 & -246.196 \\
\hline
\end{tabular}

$\mathrm{CS}_{2}$ complex for the ground and $\nu_{5}$ states of $\mathrm{CS}_{2}$. As seen in Table 2, the first bound state for the $\nu_{5}$ state is $-374.239 \mathrm{~cm}^{-1}$, which reveals the zero-point energy is $39.636 \mathrm{~cm}^{-1}$ relative to global minimum, only about one-tenth of the global well depths. Due to the deep well and high barrier for $\mathrm{Xe}-\mathrm{CS}_{2}$, the bound states are much deeper than the other $\mathrm{Rg}-\mathrm{CS}_{2}$ complexes. ${ }^{31-33,52}$ The $\nu_{1}+\nu_{3}$ band origin shift is determined by $\Delta v=E_{0}{ }^{5}-E_{0}{ }^{0}{ }^{53}$ where $E_{0}{ }^{v}$ are the ground state energies of Xe$\mathrm{CS}_{2}$ with the $\mathrm{CS}_{2}$ monomer in the corresponding $\nu$ vibrational state. Meanwhile, we further predicted the infrared band origin shift of Xe- $\mathrm{CS}_{2}$, which is $-1.996 \mathrm{~cm}^{-1}$ indicating that is negative (red-shift) in sign and large in magnitude. In addition, the band origin shifts of the other $\mathrm{Rg}-\mathrm{CS}_{2}$ complexes ${ }^{26,29-33,51,52}$ are listed in Table 3, together with the $\mathrm{Rg}-\mathrm{CO}_{2}$ complexes. ${ }^{2,6}$ One can see that the tendency of the $\mathrm{CS}_{2}$ shifts are similar to $\mathrm{CO}_{2}$. Besides, the results indicate the lighter complexes $(\mathrm{He}, \mathrm{Ne})$ tend to positive (blue shifts), while the larger complexes (Ar, Kr, Xe) tend to negative (red-shifts). The predicted $\nu_{1}+\nu_{3}$ band shift for $\mathrm{Xe}-\mathrm{CS}_{2}\left(-1.996 \mathrm{~cm}^{-1}\right)$ is larger than that for $\mathrm{Xe}^{-} \mathrm{CO}_{2}$ $\left(-1.447 \mathrm{~cm}^{-1}\right)$, revealing that Xe-CS $\mathrm{CS}_{2}$ has a larger weakening of the vdW bond upon vibrational excitation in the $\nu_{1}+\nu_{3}$ region.

In Fig. 2, we present the contour plots of the wave functions of Xe-- $\mathrm{CS}_{2}$ with $\mathrm{CS}_{2}$ at the ground state. It is clear from Fig. 2 that the vibrational ground state is localized around the T-shaped global minimum. The first two vibrational excited states are characterized predominantly by bending and stretching vibrations, respectively, while the corresponding wave functions are distributed and exhibit a strong mixing between the bending and stretching vibration modes for the higher vibrational

Table 1 The stationary geometries $\left(\AA\right.$ and deg) and well depths $\left(\mathrm{cm}^{-1}\right)$ of $\mathrm{Rg}-\mathrm{CS}_{2}(\mathrm{Rg}=\mathrm{He}, \mathrm{Ne}, \mathrm{Ar}, \mathrm{Kr}, \mathrm{Xe})$

\begin{tabular}{lllr}
\hline $\mathrm{Rg}-\mathrm{CS}_{2}$ & Global minimum & Local minimum & Saddle point \\
\hline $\mathrm{He}-\mathrm{CS}_{2}$ (ref. 31) & $(3.36,90,-52.68)$ & $(5.00,0 / 180,-32.11)$ & $(4.56,59 / 121,-21.39)$ \\
$\mathrm{Ne}-\mathrm{CS}_{2}$ (ref. 33) & $(3.44,90,-104.35)$ & $(5.03,0 / 180,-67.15)$ & $(4.56,44 / 136,-51.12)$ \\
$\mathrm{Ar}-\mathrm{CS}_{2}$ (ref. 32) & $(3.68,90,-279.77)$ & $(5.26,0 / 180,-173.47)$ & $(4.92,39 / 141,-146.75)$ \\
$\mathrm{Kr}-\mathrm{CS}_{2}$ (ref. 52) & $(3.76,90,-367.06)$ & $(5.39,0 / 180,-226.16)$ & $(4.92,45 / 135,-203.49)$ \\
$\mathrm{Xe}-\mathrm{CS}_{2}$ (ref. 51) & $(3.97,90,-414.77)$ & $(5.56,0 / 180,-243.66)$ & $(5.08,46 / 134,-224.37)$ \\
$\mathrm{Xe}-\mathrm{CS}_{2}$ & $(3.97,90,-413.88)$ & $(5.56,0 / 180,-248.04)$ & $(5.08,45 / 135,-225.88)$
\end{tabular}


Table 3 The vibrational shifts of band origin for $\mathrm{Rg}-\mathrm{CS}_{2}$, compared with $\mathrm{Rg}-\mathrm{CO}_{2}\left(\right.$ in $\mathrm{cm}^{-1}$ )

\begin{tabular}{llll}
\hline & $\mathrm{CO}_{2} \nu_{3}$ & $\mathrm{CS}_{2} \nu_{3}$ & $\mathrm{CS}_{2} \nu_{1}+\nu_{3}$ \\
\hline $\mathrm{He}$ & $+0.095($ ref. 6) & +0.108 (ref. 26) & +0.228 (ref. 31) \\
$\mathrm{Ne}$ & $+0.130($ ref. 2) & +0.180 (ref. 26) & +0.251 (ref. 33) \\
$\mathrm{Ar}$ & $-0.470($ ref. 2) & +0.067 (ref. 26) & -0.049 (ref. 32) \\
$\mathrm{Kr}$ & $-0.884($ ref. 2) & -0.787 (ref. 51) & -1.236 (ref. 52) \\
$\mathrm{Xe}$ & -1.447 (ref. 2) & -1.066 (ref. 51) & -1.996 \\
\hline
\end{tabular}

excited states. The calculated average distance $\langle R\rangle$ and the average angle $\langle\theta\rangle$ for the ground state are $3.99 \AA$ and $87.85^{\circ}$, respectively, which is very close to the global minimum $(R=$ $3.97 \AA$ and $\left.\theta=90^{\circ}\right)$. The radial dispersion $\sqrt{\left\langle R^{2}\right\rangle-\langle R\rangle^{2}}$ and angular dispersion $\sqrt{\left\langle\theta^{2}\right\rangle-\langle\theta\rangle^{2}}$ are $0.14 \AA$ and $2.15^{\circ}$, respectively. Therefore, the ground state is rigid.

The rovibrational energies of $\mathrm{Xe}-\mathrm{CS}_{2}$ are labeled by the asymmetric rotor quantum numbers $J_{\text {KaKc }}$, where $J$ denotes the total angular momentum, $K_{a}$ and $K_{c}$ represent the projections of $J$ onto the $a$ and $c$ principal axes of inertia. The rovibrational energy levels consist of four blocks, (even/even), (even/odd), (odd/even), and (odd/odd) for different combination parity of $(j / p)$. The rovibrational energies of $\mathrm{Xe}-\mathrm{CS}_{2}$ for the vibrational ground and $\nu_{1}+\nu_{3}$ excited states are shown in Table 4, and the total angular momentum $J$ ranges from 0 to 5 .

The rovibrational energy levels within $J \leq 3$ are used to fit to a Watson asymmetric top Hamiltonian ${ }^{54}$ employing the $a$-type reduction in the $I^{r}$ representation,

$$
\begin{aligned}
H= & \frac{1}{2}(B+C) J^{2}+\left[A-\frac{1}{2}(B+C)\right] J_{a}{ }^{2}+\frac{1}{2}(B-C)\left(J_{b}{ }^{2}-J_{c}{ }^{2}\right) \\
& -\Delta_{J} J^{4}-\Delta_{J K} J_{a}{ }^{2} J^{2}-\Delta_{K} J_{a}{ }^{4}-2 \delta_{J} J^{2}\left(J_{b}{ }^{2}-J_{c}{ }^{2}\right) \\
& -\delta_{K}\left[J_{a}{ }^{2}\left(J_{b}{ }^{2}-J_{c}{ }^{2}\right)+\left(J_{b}{ }^{2}-J_{c}{ }^{2}\right) J_{a}{ }^{2}\right]
\end{aligned}
$$

The fitted molecular spectroscopic constants for the Xe- $\mathrm{CS}_{2}$ complex are given in Table 5. Because the energy levels for Xe$\mathrm{CS}_{2}$ at the ground state are almost equal to the $\nu_{1}+\nu_{3}$ excited state, the molecular parameters of the two states are very similar. The calculated inertial defects $\Delta_{0}$ of this complex with $\mathrm{CS}_{2}$ at the ground state and the $\nu_{1}+\nu_{3}$ excited state are around $1.07 \mathrm{amu} \AA^{2}$ and $1.13 \mathrm{amu} \AA^{2}$, respectively. For $\mathrm{Rg}-\mathrm{CS}_{2}(\mathrm{Rg}=$ $\mathrm{He},{ }^{31} \mathrm{Ne},{ }^{33} \mathrm{Ar}^{32} \mathrm{Kr}^{52}$ ), the inertial defects $\Delta_{0}$ in the ground state

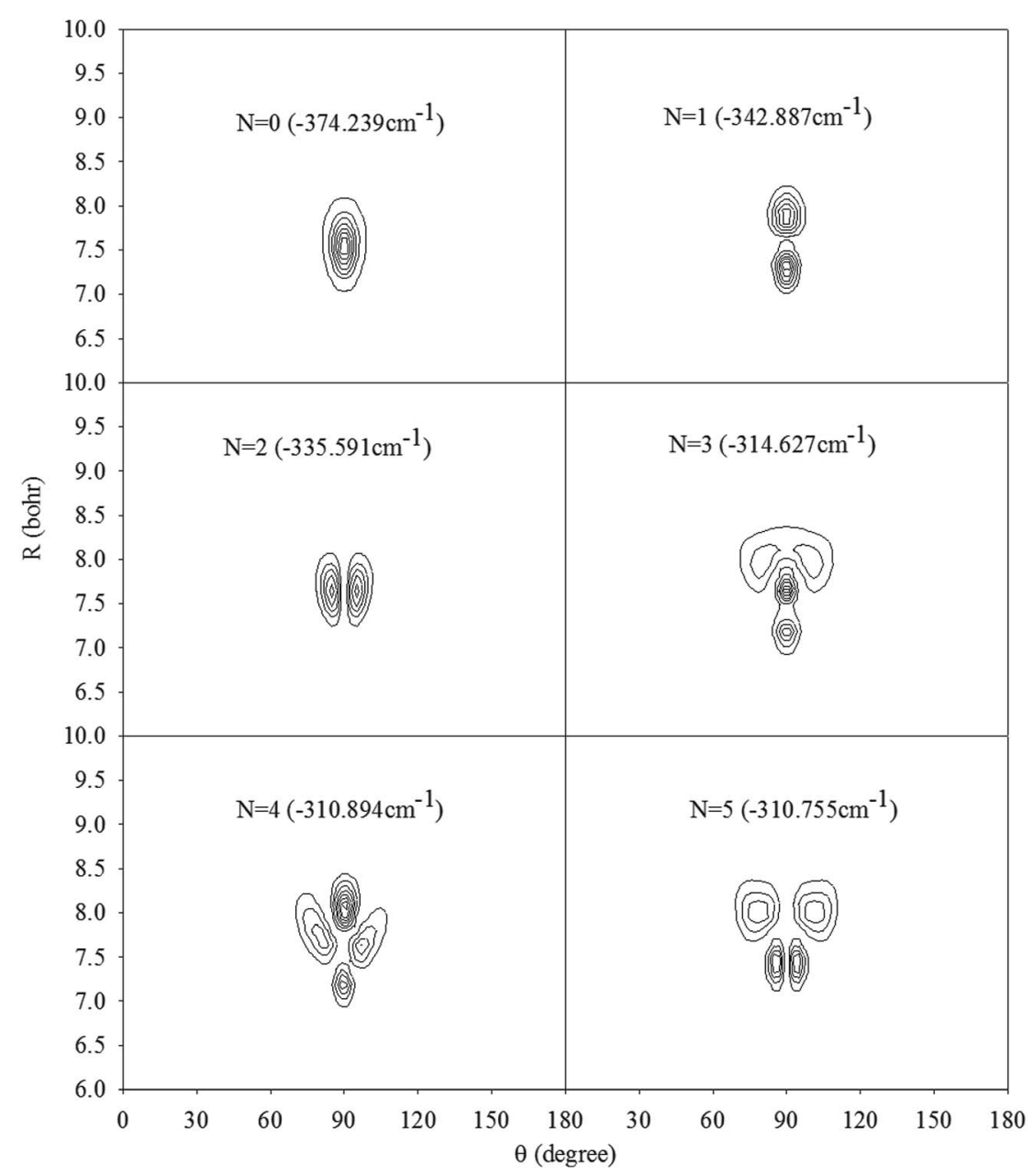

Fig. 2 Contour plots of the wave functions for the lowest six vibrational states of $\mathrm{Xe}-\mathrm{CS}_{2}$ with $\mathrm{CS}_{2}$ at the $\nu_{1}+\nu_{3}$ excited state. 
Table 4 The rovibrational energy levels (in $\mathrm{cm}^{-1}$ ) of $\mathrm{Xe}-\mathrm{CS}_{2}$ with $\mathrm{CS}_{2}$ at the ground and $\nu_{1}+\nu_{3}$ excited states

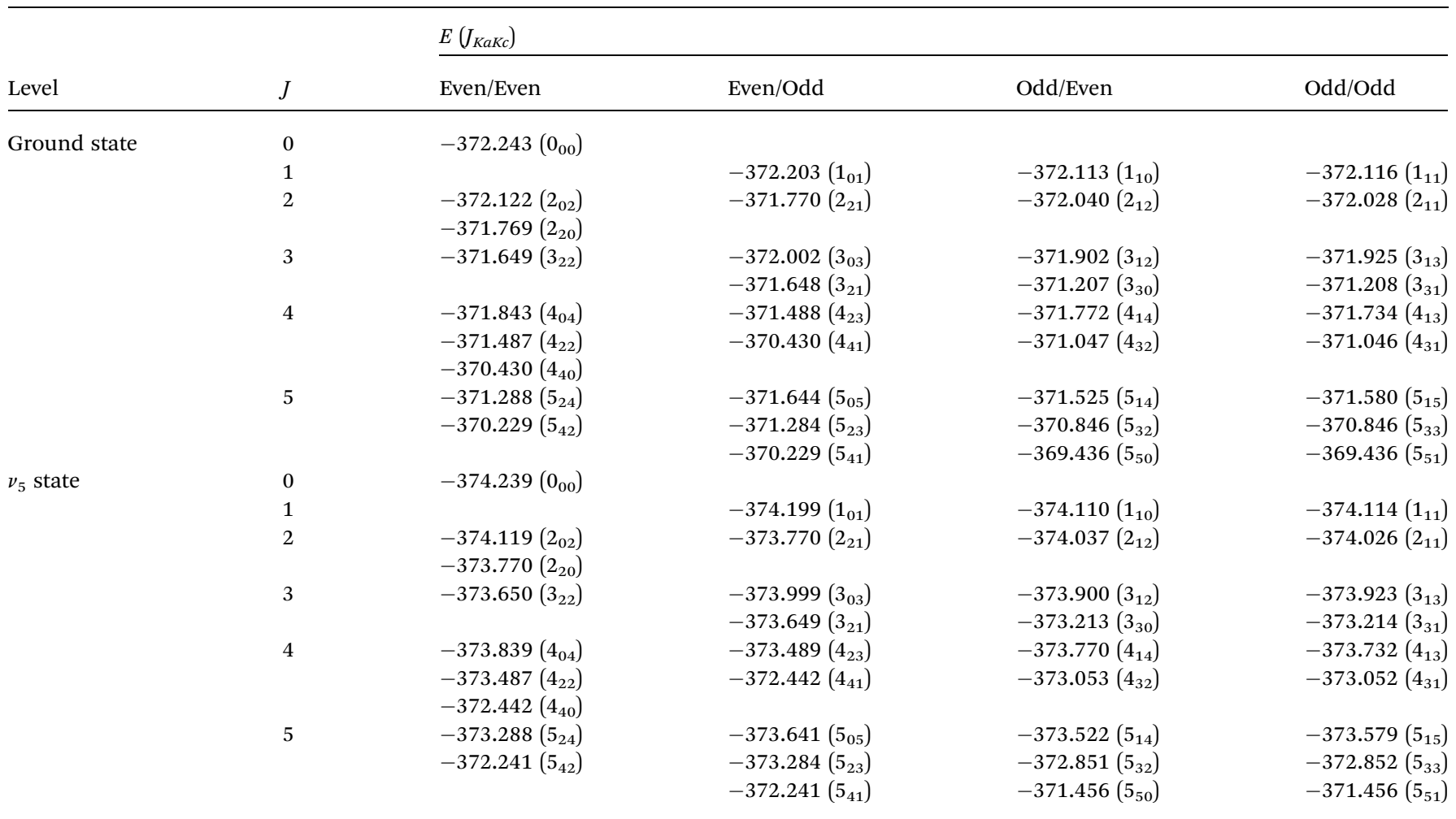

Table 5 The calculated spectroscopic constants (in $\mathrm{cm}^{-1}$ ) and the inertial defects $\Delta_{0}$ (in amu $\AA^{2}$ ) for the $\mathrm{Xe}-\mathrm{CS}_{2}$ complex with $\mathrm{CS}_{2}$ at the ground and the $\nu_{1}+\nu_{3}$ excited states

\begin{tabular}{lll}
\hline & Ground state & $\nu_{5}$ state \\
\hline$A$ & 0.108321 & 0.107674 \\
$B$ & 0.022038 & 0.022023 \\
$C$ & 0.018291 & 0.018261 \\
$\Delta_{K}$ & $-2.443 \times 10^{-7}$ & $-3.110 \times 10^{-7}$ \\
$\Delta_{J K}$ & $3.748 \times 10^{-7}$ & $3.258 \times 10^{-7}$ \\
$\Delta_{J}$ & $4.563 \times 10^{-6}$ & $8.778 \times 10^{-6}$ \\
$\delta_{K}$ & $2.050 \times 10^{-6}$ & $2.225 \times 10^{-6}$ \\
$\delta_{J}$ & $9.176 \times 10^{-9}$ & $2.993 \times 10^{-9}$ \\
$\Delta_{0}$ & 1.07 & 1.13 \\
& &
\end{tabular}

were found to be $6.14,3.09,2.93,2.32$ amu $\AA^{2}$, respectively. The decrease in the inertial defects reveals that the vdW complexes are more rigid with the increase in the mass of the rare gas atom. The asymmetry parameter $\kappa, \kappa=(2 \mathrm{~B}-\mathrm{A}-\mathrm{C}) /(\mathrm{A}-\mathrm{C})$ (equal to -1 for the symmetric prolate top) is -0.917 and -0.916 for this complex with $\mathrm{CS}_{2}$ at the ground and the $\nu_{1}+\nu_{3}$ excited states, respectively.

\section{Conclusions}

In this paper, a new four-dimensional potential energy surface for the Xe-CS $\mathrm{CS}_{2}$ complex including the $Q_{1}$ and $Q_{3}$ normal modes was constructed. We calculated the intermolecular potential with five PODVR grid points for the $Q_{1}$ and $Q_{3}$ normal modes, respectively, using the $\operatorname{CCSD}(\mathrm{T})$ method with the aug-cc-pVQZ basis set plus bond functions. Based on the $a b$ initio potential points, two vibrationally averaged PESs of the $\mathrm{Xe}-\mathrm{CS}_{2}$ complex were generated. We found that each potential energy surface has a T-shaped global minimum and two equivalent local linear minima. The bound rovibrational energy levels of $\mathrm{Xe}-\mathrm{CS}_{2}$ were obtained by employing the radial DVR/angular FBR method and the Lanczos algorithm. The predicted band origin shift is $-1.996 \mathrm{~cm}^{-1}$. Meanwhile, we found that the $\mathrm{Rg}-\mathrm{CS}_{2}$ complexes have very similar features. For example, each PES is characterized by a T-shaped global minimum. For another, the regularities were represented among the complexes from $\mathrm{He}-\mathrm{CS}_{2}$ to $\mathrm{Xe}-\mathrm{CS}_{2}$. It is expected that the work on the Rg-CS${ }_{2}$ complexes in the $\nu_{1}+\nu_{3}$ region of $\mathrm{CS}_{2}$ should be useful for further theoretical and experimental studies.

\section{Conflicts of interest}

There are no conflicts to declare.

\section{Acknowledgements}

This work was supported by the National Natural Science Foundation of China (Grant No. 21705011).

\section{References}

1 G. T. Fraser, A. S. Pine and R. D. Suenram, J. Chem. Phys, 1988, 88, 6157.

2 R. W. Randall, M. A. Walsh and B. J. Howard, Faraday Discuss. Chem. Soc., 1988, 85, 13. 
3 M. Iida, Y. Ohsbima and Y. Endo, J. Phys. Chem., 1993, 97, 357.

4 A. S. Pine and G. T. Fraser, J. Chem. Phys., 1988, 89, 100.

5 T. Konno, S. Fukuda and Y. Ozaki, Chem. Phys. Lett., 2006, 421, 421.

6 M. J. Weida, J. M. Sperhac and D. J. Nesbitt, J. Chem. Phys., 1994, 101, 8351.

7 Y. J. Xu and W. Jäger, J. Mol. Spectrosc., 1998, 192, 435.

8 G. A. Parker, M. Keil and A. Kuppermann, J. Chem. Phys., 1983, 78, 1145.

9 M. Keil and G. A. Parker, J. Chem. Phys., 1985, 82, 1947.

10 L. Beneventi, P. Casavecchia, F. Vecchiocattivi, G. G. Volpi, U. Buck, C. Lauenstein and R. Schinke, J. Chem. Phys., 1988, 89, 4671.

11 C. F. Roche, A. Ernesti, J. M. Huston and A. S. Dickinson, J. Chem. Phys., 1996, 104, 2156.

12 P. J. Marshall, M. M. Szczesniak, J. Sadlej, G. Chalasinski, M. A. ter Horst and C. J. Jameson, J. Chem. Phys., 1996, 104, 6569.

13 J. M. Hutson, A. Ernesti, M. M. Law, C. F. Roche and R. J. Wheatley, J. Chem. Phys., 1996, 105, 9130.

14 G. S. Yan, M. H. Yang and D. Q. Xie, J. Chem. Phys., 1998, 109, 10284.

15 F. Negri, F. Ancliotto, G. Mistura and F. Toigo, J. Chem. Phys., 1999, 111, 6439.

16 H. Ran and D. Q. Xie, J. Chem. Phys., 2008, 128, 124323.

17 Y. L. Cui, H. Ran and D. Q. Xie, J. Chem. Phys., 2009, 130, 224311.

18 R. Chen, E. Q. Jiao, H. Zhu and D. Q. Xie, J. Chem. Phys., 2010, 133, 104302.

19 R. Chen and H. Zhu, J. Theor. Comput. Chem., 2012, 11, 1175.

20 R. Chen, H. Zhu and D. Q. Xie, Chem. Phys. Lett., 2011, 511, 229.

21 M. Chen and H. Zhu, J. Theor. Comput. Chem., 2012, 11, 537.

22 H. Li and R. J. Le Roy, Phys. Chem. Chem. Phys., 2008, 10, 4128.

23 H. Li, N. Blinov, P.-N. Roy and R. J. Le Roy, J. Chem. Phys., 2009, 130, 144305.

24 Y. L. Cui, H. Ran and D. Q. Xie, J. Theor. Comput. Chem., 2008, 7, 707.

25 C. J. Xie, R. Chen, H. Zhu and D. Q. Xie, Chem. Res. Chin. Univ., 2009, 9, 1851.

26 F. Mivehvar, C. Lauzin, A. R. W. McKellar and N. MoazzenAhmadi, J. Mol. Spectrosc., 2012, 281, 24.
27 H. Farrokhpour and M. Tozihi, Mol. Phys., 2013, 111, 779.

28 L. M. Zang, W. Dai, L. M. Zheng, C. X. Duan, Y. P. Lu and M. H. Yang, J. Chem. Phys., 2014, 140, 114310.

29 T. Yuan and H. Zhu, Theor. Chem. Acc., 2014, 133, 1537.

30 T. Yuan, X. L. Sun, Y. Hu and H. Zhu, J. Chem. Phys., 2014, 141, 104306.

31 J. Shang, T. Yuan and H. Zhu, Theor. Chem. Acc., 2016, 135, 1.

32 J. Shang, T. Yuan and H. Zhu, Chem. Phys. Lett., 2016, 648, 147.

33 M. Qin, J. Shang, Q. Hong and H. Zhu, Mol. Phys., 2017, 115, 379.

34 J. S. Wells, M. Schneider and A. G. Maki, J. Mol. Spectrosc., 1988, 132, 422.

35 J. M. Bowman and B. Gazdy, J. Chem. Phys., 1991, 94, 816.

36 H. Wei and T. Carrington, J. Chem. Phys., 1992, 97, 3029.

37 J. Echave and D. C. Clary, Chem. Phys. Lett., 1992, 190, 225.

38 M. Meuwly and J. M. Hutson, J. Chem. Phys., 2003, 119, 8873.

39 D. E. Woon and T. H. Dunning, J. Chem. Phys., 1993, 98, 1358.

40 K. A. Peterson, D. Figgen, E. Goll, H. Stoll and M. Dolg, J. Chem. Phys., 2003, 119, 11113.

41 T. B. Pedersen, B. Fernandez, H. Koch and J. Makarewicz, J. Chem. Phys., 2001, 115, 8431.

42 S. F. Boys and F. Bernardi, Mol. Phys., 1970, 19, 553.

43 H. J. Werner, P. J. Knowles, R. D. Amos, A. Berning, D. L. Cooper, M. J. O. Deegan, A. J. Dobbyn, F. Eckert, S. T. Elbert, C. Hampel, R. Lindh, A. W. Lloyd, W. Meyer, A. Nicklass, K. Peterson, R. Pitzer, A. J. Stone, P. R. Taylor, M. E. Mura, P. Pulay, M. Schutz, H. Stoll and T. Thoorstcinsso, Molpro, version 2000.1, a package of $a b$ initio programs, 2000, see http://www.molpro.net.

44 J. Tennyson and B. T. Sutcliffe, Mol. Phys., 1984, 51, 887.

45 S. Miller and J. Tennyson, J. Mol. Spectrosc., 1988, 128, 530.

46 S. Y. Lin and H. Guo, J. Chem. Phys., 2002, 117, 5183.

47 R. Q. Chen, G. B. Ma and H. Guo, Chem. Phys. Lett., 2000, 320, 567.

48 D. T. Colbert and W. H. Miller, J. Chem. Phys., 1992, 96, 1982. 49 C. J. Lanczos, J. Res. Natl. Bur. Stand., 1950, 45, 255.

50 H. G. Yu, J. Chem. Phys., 2002, 117, 8190.

51 T. Yuan, M. L. Yang and H. Zhu, Comput. Theor. Chem., 2015, 88, 1070.

52 Q. Hong, M. Qin and H. Zhu, Acta Chim. Sin., 2018, 76, 138. 53 F. Paesani and K. B. Whaley, J. Chem. Phys., 2004, 121, 4180. 54 J. K. G. Watson, J. Chem. Phys., 1967, 46, 1935. 